\title{
Possibilités d'action publique pour une meilleure prise en compte des marchés domestiques du bois dans le cadre des accords de partenariat volontaire (APV) au Gabon
}

\author{
Guillaume Lescuyer, Saturnin Ndotit, Laurentine Bilogo bi Ndong, Raphaël Tsanga et Paolo Omar Cerutti
}

\section{Points essentiels}

- L'Accord sur l'application des réglementations forestières, la gouvernance et les échanges commerciaux (FLEGT) a été lancé en 2003 par l'Union européenne (UE) afin de lutter au niveau mondial contre l'exploitation forestière illégale. En particulier, le FLEGT vise à réduire le commerce du bois récolté illégalement entre l'UE et les pays partenaires producteurs de bois.

- Le FLEGT fonctionne grâce à deux principaux instruments : des accords commerciaux bilatéraux — appelés accords de partenariat volontaire (APV) - qui sont signés avec les pays producteurs qui le souhaitent et le règlement sur le bois de I'Union européenne qui est entré en vigueur en mars 2013. Ce règlement sur le bois de l'UE impose aux importateurs européens de faire preuve d'une diligence raisonnable lorsqu'ils s'approvisionnent en bois à l'étranger pour se prémunir contre les produits illégaux.

- À ce jour, six pays ont signé un APV. Cinq d'entre eux se sont même engagés à appliquer les dispositions de cet APV concernant la vérification de la légalité non seulement au bois exporté vers l'Europe, mais aussi à celui qui est commercialisé sur leurs marchés intérieurs. Ceci signifie que le bois récolté et commercialisé sur le marché domestique sera réglementé par des programmes de permis nationaux dans le cadre de l'APV (c'est le système de garantie de la légalité du bois ou Timber Legality Assurance System, TLAS).

- Le Gabon a officiellement entamé des négociations APV en septembre 2010. L'engagement initial s'est traduit par un accord sur une feuille de route et la réalisation d'une grille de légalité. Cependant, cette dernière n'a pas encore été validée et les négociations n'ont repris que récemment. Il est difficile de prédire le champ couvert par l'APV au final, mais les informations dont on dispose laissent penser qu'il concernera aussi le marché domestique.

- Le marché domestique du bois s'est transformé radicalement ces dernières années. Selon les résultats de Pro-Formal, la consommation intérieure de sciages artisanaux s'est réduite pratiquement de moitié entre 2008 et 2012 et les rebuts industriels fournissent maintenant plus de bois que par le passé, car la production industrielle pour le marché national a enregistré une hausse au cours de la même période. Ces tendances s'expliquent en grande partie par la crise financière, l'interdiction d'exporter les grumes à partir de 2010 qui a forcé les sociétés industrielles à transformer le bois localement, et des efforts accrus de contrôle et d'application de la loi.

- Ces dernières années, le ministère de l'Économie forestière (MEF) a aussi délivré un plus grand nombre de permis d'exploiter à petite échelle, y compris : des permis spéciaux, des autorisations de sciage de long (ASL) et des autorisations spéciales de coupe (ASC). Malheureusement, la plupart de ces permis ne figurent pas dans la loi forestière actuelle et prêteront à controverse. En revanche, le permis de gré à gré (PGG), seul permis destiné à l'exploitation à petite échelle et inscrit dans la loi actuelle, a été suspendu en 2010 et n'a été remis en vigueur que mi-2014.

- Les premières forêts communautaires, prévues depuis longtemps comme solution de rechange légale pour que les scieurs artisanaux s'y approvisionnent en bois, n'ont été créées qu'au début de 2013. Les permis de gré à gré et les forêts communautaires sont complémentaires et leur mise en œuvre effective pourrait fournir dans la légalité une quantité suffisante de sciages artisanaux pour le marché domestique gabonais. Cette mise en œuvre effective nécessitera un effort concerté de l'administration.

\section{Contexte}

Le secteur forestier au Gabon est régi par le code forestier qui date de 2001.' Ce secteur représente environ 4,3\% du PIB et près de $5 \%$ des exportations. II constitue le premier employeur industriel après l'État, avec presque 13000 emplois formels et au moins 1000 emplois informels (de Wasseige et al. 2009, Lescuyer et al. 2011). Ce code énumère quatre types de permis : CFAD (concession forestière sous aménagement durable), PFA (permis forestier associé), forêts communautaires et le PGG (permis de gré à gré).

À l'instar des pays du bassin du Congo, le cadre juridique au Gabon ne favorise guère le développement d'un marché domestique du bois légal. Les échanges divers qui ont eu lieu entre les représentants du projet Pro-Formal, ses partenaires gabonais et le gouvernement permettent de constater que ce 


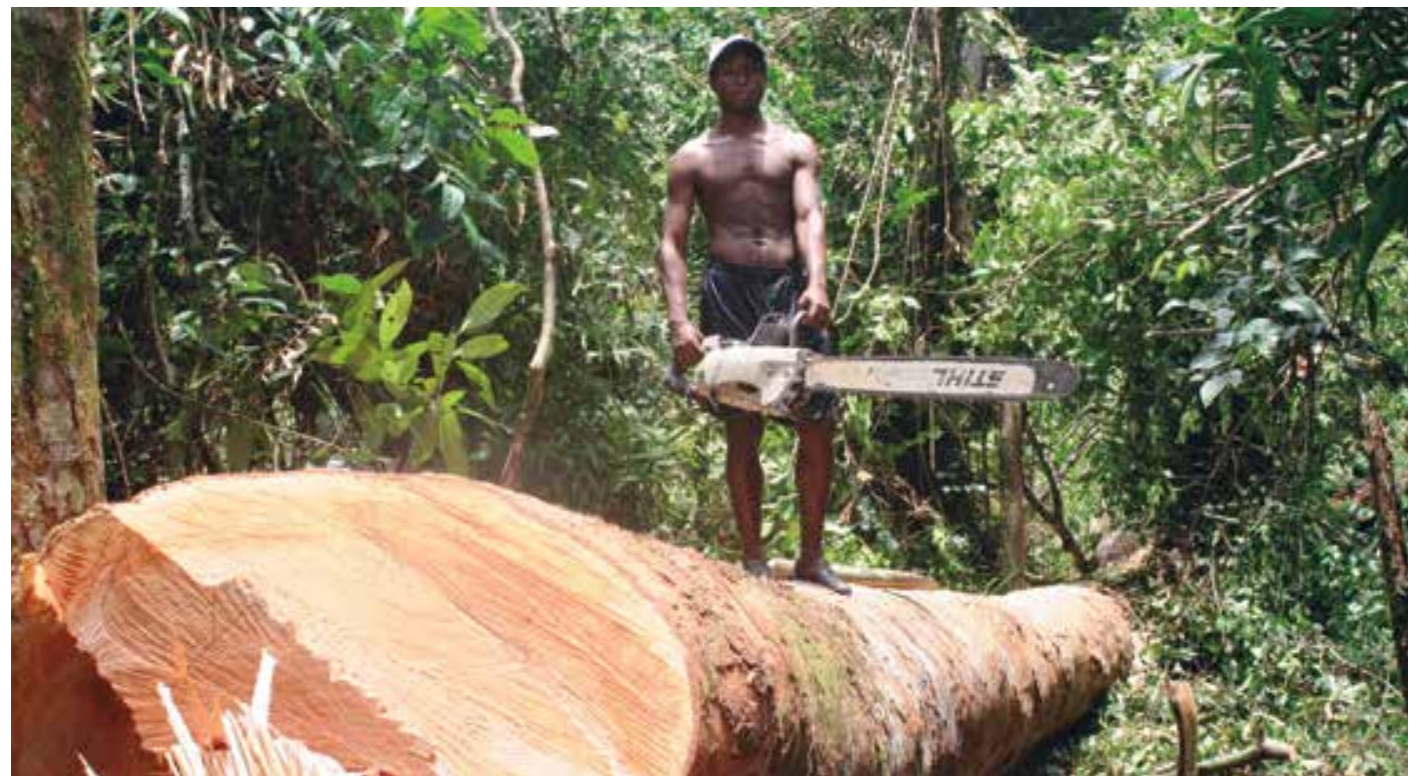

\section{Opérations de récolte}

Photo Guillaume Lescuyer, CIFOR

dernier est conscient de la nécessité de mieux tenir compte de la contribution du secteur dans l'économie nationale. Cependant, les efforts officiels pour mieux comprendre, réglementer et contrôler le secteur sont encore insuffisants. Par exemple, aucune donnée officielle sur la production et la vente n'est recueillie.

Le Gabon présente cependant au moins deux grandes différences avec les autres pays de la région en ce qui concerne le sciage artisanal et les marchés domestiques du bois. D'abord, la grande majorité de la population vit dans la capitale, Libreville, où la consommation de bois se concentre également (Lescuyer et al. 2011). Comparativement à d'autres pays, cette situation pourrait faciliter la mise en œuvre de futures mesures ciblant l'aval de la

\section{Tableau 1. Principales conclusions quantitatives - Gabon}

\begin{tabular}{lrr}
\hline Sciages (m³/an) & 2008-2009 & 2011-2012 \\
\hline $\begin{array}{l}\text { Libreville (ventes enregistrées } \\
\text { sur les marchés) }\end{array}$ & 51100 & 22382 \\
\hline $\begin{array}{l}\text { Libreville (entrées dans la ville } \\
\text { par la route) }\end{array}$ & 60000 & 32300 \\
\hline
\end{tabular}

\begin{tabular}{lll}
\hline Production industrielle & $280000 \quad 439000$
\end{tabular}

\begin{tabular}{lcc}
\hline $\begin{array}{l}\text { Personnes employées par le } \\
\text { secteur informel du bois (villes) }\end{array}$ & 1000 & 504 \\
\hline $\begin{array}{l}\text { Contribution à l'économie } \\
\text { locale (en millions EUR) }\end{array}$ & 2,7 & 1,6 \\
\hline Bénéfice par m m $^{3}$ exploité (EUR) & 36,7 & 18,6 \\
\hline Marge bénéficiaire & $32 \%$ & $14 \%$ \\
\hline
\end{tabular}

Sources : Lescuyer et al. 2011 ; Ministère de l'Économie forestière (MEF) 2012. chaîne du bois. Deuxièmement, les résultats montrent que la consommation domestique a été pratiquement divisée par deux entre 2008 et 2012 (Tableau 1). Les résultats de 2012, qui ont été collectés à l'échelle nationale, mettent aussi en évidence une baisse du bénéfice moyen par rapport aux données de 2009 qui ne portaient que sur la région de Libreville.

Plusieurs facteurs pourraient expliquer cette tendance. D'abord, une interdiction d'exporter les grumes a été annoncée par décret présidentiel en novembre 2009 (avec entrée en vigueur début 2010, repoussée à mi-2010). Cette interdiction a pris au dépourvu le secteur du bois qui a dû prendre rapidement des mesures pour transformer $100 \%$ de sa production au Gabon. Cette décision a par conséquent accru la quantité de sciages produits localement, avec un impact direct sur les volumes de rebuts de scierie (non exportés) disponibles pour le marché domestique du bois. Certaines entreprises forestières qui exportaient des grumes avant l'interdiction, se retrouvaient aussi obligées de transformer ces grumes et de les vendre sur le marché domestique comme sciages, car elles ne disposaient pas encore d'une clientèle internationale pour les sciages. Ceci ne pourrait être cependant qu'une source de bois à court terme pour le marché domestique, car les entreprises mettent en place de nouveaux canaux pour vendre leurs sciages. Deuxièmement, les données recueillies sur la période 2008-2009 sont sans doute inhabituelles, car de nombreux grands projets d'infrastructure (p. ex. routes, ponts et stades) ont été réalisés pour accueillir la Coupe d'Afrique des Nations qui a eu lieu en 2010. À cet égard, la consommation en 2011-2012 pourrait être plus représentative de la demande sur le marché domestique en temps normal. Troisièmement, les entretiens menés par l'équipe du projet semblent indiquer que le MEF a récemment accru ses efforts de contrôle et d'application de la loi, peut-être sous l'effet du regain d'attention que la présidence porte à l'environnement.

Sur la même période, le MEF a émis un plus grand nombre de permis destinés aux petits exploitants. En 2008, environ $79 \%$ des scieurs artisanaux ne disposaient pas de permis de coupe. En 


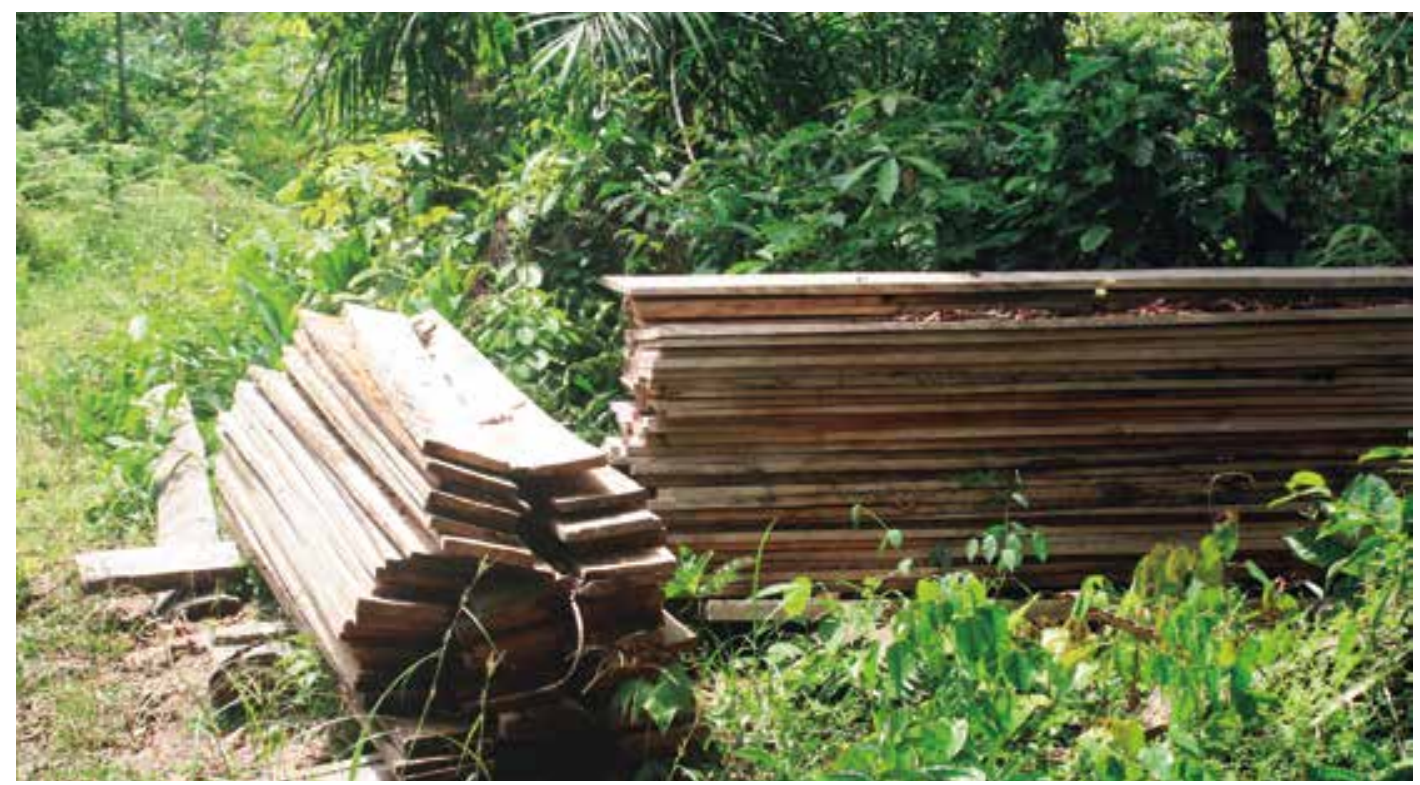

\section{Bois prêt à être transporté dans la forêt aux marchés de la ville}

Photo Guillaume Lescuyer, CIFOR

2012, ce chiffre était tombé à $58 \%$. Cela peut révéler un effort en vue de permettre aux petits opérateurs d'accéder légalement au bois. Malheureusement, la plupart des permis délivrés récemment ne figurent pas dans la loi actuelle.

En revanche, le permis de gré à gré (PGG), seul permis destiné à l'exploitation à petite échelle et inscrit dans la loi actuelle, a été suspendu en 2010 et n'a été remis en vigueur que mi-2014. Les premières forêts communautaires, prévues depuis longtemps comme solution de rechange légale pour les scieurs artisanaux, ont été créées au début de 2013. Leur nombre officiel pourrait augmenter et une mise en œuvre effective pourrait suivre sous peu, mais cela nécessitera un effort concerté de la part de l'administration. Cette question devrait faire partie des négociations APV ou de toute discussion visant l'amélioration du cadre juridique actuel, ainsi que la prise en compte totale, ou l'exclusion définitive, des permis accordés en ce moment.

\section{Possibilités d'action}

Problème Plusieurs permis sont actuellement délivrés pour le sciage artisanal « légal ».

\section{Recommandations}

- $\quad$ Selon les résultats, les permis qui conviendraient le mieux sont I'ASC et l'ASL. Seuls ces deux permis devraient être délivrés à long terme, après clarifications et amendements à la réglementation actuelle, comme cela a été initiée en juin 2014. En particulier, comme dans le cas des autres pays du bassin du Congo, la décentralisation de la délivrance, du suivi et du recours aux sanctions semblent les meilleurs moyens d'éviter d'interrompre l'approvisionnement du marché domestique (au moins à court ou moyen terme) par l'introduction du cadre centralisé de l'APV.

- Une réglementation est nécessaire pour clarifier plusieurs problèmes actuels liés à l'ASC, notamment la question de savoir s'il doit aussi concerner le droit d'usage coutumier qui ne doit pas s'exercer actuellement dans un but lucratif.
- Les permis spéciaux ne devraient plus être délivrés, car ils ne constituent pas un permis de coupe officiel en vertu du code forestier.

- Le nombre de forêts communautaires pilotes devrait être augmenté pour promouvoir l'exploitation légale à petite échelle susceptible de concurrencer le sciage artisanal informel.

\section{Répercussions sur l'économie rurale}

Problème II n'existe pas d'« agrément » officiel ou de document qui accorde le statut de « scieur artisanal ».

\section{Recommandation}

Créer un « agrément » ou une reconnaissance officielle similaire pour les scieurs artisanaux professionnels, lequel serait renouvelé tous les ans ou tous les deux ans en contrepartie d'une taxe modique. Cet agrément devrait exister avant l'attribution des futurs permis.

Problème Les résultats permettent de constater que de nombreux conflits se posent dans les zones rurales parce que la population a besoin de scieurs artisanaux (ainsi que d'entreprises forestières industrielles) pour fournir une infrastructure que ces derniers sont incapables d'offrir à cause d'un manque de moyens financiers et techniques adéquats.

\section{Recommandation}

- La législation et la réglementation remaniées sur les ASL pourraient envisager les agréments avec les propriétaires coutumiers (aussi signés par ces derniers) comme un prérequis à l'attribution de permis.

\section{Gouvernance}

Problème On constate une prolifération de barrages routiers qui accentuent la pression financière sur les activités forestières de plusieurs ministères. 


\section{Recommandation}

Étant donné la situation particulière de Libreville comme lieu de consommation final, les barrages routiers pourraient être organisés conjointement par plusieurs ministères et situés en des points d'entrée stratégiques où des taxes légalement instituées seraient collectées.

Problème Le système actuel de «primes » pour l'application de la loi n'est fait que pour récompenser les fonctionnaires de haut rang au lieu de ceux qui sont sur le terrain.

\section{Recommandation}

- Réorganiser le système actuel de « primes » pour l'application de la loi afin qu'une part supérieure de celleci revienne à l'équipe ou au fonctionnaire qui décèle l'infraction et établit le procès-verbal.

Problème De nombreux scieurs artisanaux (individuels ou en groupe) peuvent poursuivre leur activité actuelle parce qu'ils sont protégés par des fonctionnaires de haut rang.

\section{Recommandation}

- Comme étape préliminaire, les infractions et sanctions éventuelles pourraient être rendues publiques de manière régulière par le MEF (au moyen d'une publication trimestrielle dans les quotidiens et de communiqués de presse sur les stations de radio locales) et par les organisations non gouvernementales (sur leur site internet), en vue de décourager les écarts de conduite des agents de l'État.

\section{Bibliographie}

de Wasseige C, Devers D, de Marcken P, Eba'a Atyi R, Nasi R et Mayaux P, éds. 2009 Les Forêts du Bassin du Congo - État des Forêts 2008. Office des publications de l'Union Européenne, Kinshasa.

Lescuyer G, Cerutti PO, Ndotit Manguiengha S et Bilogo bi Ndong L. 2011 The domestic market for small-scale chainsaw milling in Gabon: Present situation, opportunities and challenges. Document occasionnel 65. Centre de recherche forestière internationale, Bogor, Indonésie. [Version française aussi disponible : Lescuyer G, Cerutti PO, Ndotit Manguiengha S et Bilogo bi Ndong L. 2011 Le marché domestique du sciage artisanal à Libreville, Gabon : État des lieux, opportunités et défis. Document occasionnel 63. Centre de recherche forestière internationale, Bogor, Indonésie.]

\section{Lectures complémentaires}

Kishor N et Lescuyer G. 2012 Controlling illegal logging in domestic and international markets by harnessing multilevel governance opportunities. International Journal of the Commons 6(2):255-70.

Putzel L, Kelly A, Cerutti PO et Artati Y. 2014 Formalization of natural resource access and trade: Insights from land tenure, mining, fisheries, and non-timber forest products. Centre de recherche forestière internationale, Bogor, Indonésie.

Wit M, van Dam J, Cerutti PO, Lescuyer G, Kerrett R et Parker Mckeon J. 2011 Chainsaw milling: Supplier to local markets - A synthesis. Dans : Wit M et van Dam J, éds. Chainsaw milling: Supplier to local markets. Tropenbos International, Wageningen, Pays-Bas. VII-XXII.

Le projet Pro-Formal (EuropeAid/ENV/2010-242904/TPS), financé par I'UE, a été mis en œuvre par le Centre de recherche forestière internationale (CIFOR) dans trois régions (Asie du Sud-Est, Afrique subsaharienne et Amérique latine) et cinq pays (Indonésie, Cameroun, République démocratique du Congo, Gabon et Équateur) de juillet 2010 à décembre 2013. Le projet s'est déroulé avec plusieurs partenaires clés, et certains résultats ont déjà été diffusés par le CIFOR grâce à ses documents occasionnels, Infobriefs, articles publiés dans des revues scientifiques et autres articles sur internet (consultables sur le site internet du projet : www.cifor.org/pro-formal).

\begin{tabular}{l|l} 
RESEARCH \\
PROGRAM on \\
Forests, Trees and
\end{tabular}

Cette recherche a été menée par le CIFOR dans le cadre du Programme de recherche du CGIAR sur les forêts, les arbres et l'agroforesterie (CRP-FTA). Ce programme collaboratif vise à améliorer la gestion et l'utilisation des forêts, de l'agroforesterie et des ressources génétiques des arbres à l'échelle du paysage, des forêts aux exploitations agricoles. Le CIFOR dirige le CRP-FTA en partenariat avec Bioversity International, le CATIE, le CIRAD, le Centre international d'agriculture tropicale et le Centre mondial de l'Agroforestrie.

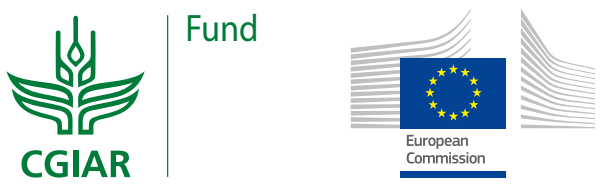

